\title{
Diving Health: Principles of medical \& physical fitness
}

\author{
Authors \\ Ibrahim A Albrethen, Ali M Aldossary, Ahmad A Alghamdi, Hussein M Alkahtani, Munairah A \\ Alswilem, Abdullah M Alramzi, Ali A Ablowi, Hani A Alkhudhier, Ali A Alqarni
}

\section{Introduction}

The health risks of deep diving and the fitness of divers continue to attract the attention of researchers. Increased deep diving for commercial, military, and recreational purposes and the persistence of existential threats to divers in the underwater, hyperbaric environment, underscore sustained interest in diving health and improving treatments for health disorders associated with diving.

In the article "Exercise Effects During Diving and decompression on Postdive Venous Gas Embolism" Jankowski, Tikuisis, and Nishi (2004) report on their study, in which they pursued an emerging challenge to the conventional view that exercise during diving is entirely a contributor to two well known risks in diving, decompression sickness (DCS) and venous gas embolism (VGE). Their research objective was to find evidence that a certain exercise regimen for divers could minimise VGE events and concomitantly DCS.

In another study based article "Pulmonary Function in Military Divers: Smoking Habits and Physical Fitness Training Influence," Sekulic and Tocilj (2006) investigated the impact of physical fitness training among smoking and non-smoking divers.

As a follow-up of a review of the aforementioned articles, we discuss some of the published opinions on diving health and diving diseases. This discussion proceeds in 5 sections:

1) The study by Jankowski, Tikuisis, and Nishi.

2) Diving and Divers' Health.

3) Issues of concern in a medical assessment of divers.

4) The study by Sekulic and Tocilj.

5) Discussion

\section{The Jankowski, Tikuisis and Nishi study}

The hypothesis that Jankowski, Tikuisis and Nishi (2004) investigated was that "bubble activity observed at both the precordium and subclavian vein sites would be reduced if moderate exercise were performed intermittently during decompresssion" (pp.489, 490). In this respect, these researchers followed up studies, which had investigated the implications of moderate exercise, at intervals during the ascent phase of diving, on severe events of gas bubbles in the vascular system of divers. Typically, these bubbles of gas derive from inhalation of breathing gas mixtures by divers during their underwater experience.

In their experiment, Jankowski, Tikuisis and Nishi used a methodology of simulated dives by the subjects. This activity took place in a water filled hyperbaric chamber [a compressed air diving chamber], where each dive was to a pressure of 450 
kPA and had 30 minutes duration. Doppler ultrasonic bubble detectors were used to monitor VGE in the participants. According to D'Aoust et al. (1977) Doppler ultrasound is routine technique in hyperbaric physiology. Its measurement principle uses the frequency of reflected ultrasound from moving intravascular bubbles (Daniels, Evans, Hennessy and Estaugh, 1984).

The value of Jankowski, Tikuisis and Nishi's study is its addition to the development of knowledge that could reduce the incidence of DCS, a prime hazard of diving. For instance, their investigation suggested that exercise during the decompression phase of diving could facilitate efficient elimination of inert gas from the tissues, thus averting VGE and DCS.

In the literature of Vann, Butler, Mitchell, and Moon (2011, p.153), DCS is induced by a "state of super-saturation," in which there is an accumulation of inert gas in tissues, over and above the level of ambient or environment pressure; this development allows inert gas to flow from the tissues. In the deep diving context, inert gases, mostly helium and nitrogen, are components of the breathing mixture for divers.

DCS is visible in bubbles in blood or tissue. According to Strauss and Borer (2001), in every theory of DCS, bubbles are a primary contributory factor. Strauss and Borer (2001) also point out that DCS and arterial gas embolism (AGE), of which VGE is a subset, are intertwined so closely that it is often difficult to segregate these conditions. DeGorordo, Vallejo-Manzur, Chanin and Varon (2003, p.175) distinguish two categories in the pathophysiology of DCS: type 1 and type 2 DCS. Type 1 DCS is sometimes called the 'bends'; its main manifestations are "deep, aching and poorly localized musculoskeletal pain." Usually, type 1 DCS develops within one hour of a diver's return to the surface and its manifestations, which may commence mildly, intensify gradually over the next twenty-four to thirty-six hours. Bubble formation in the skin and lymphatics causes rash and lymphoedema during type 1 DCS. In type 2 DCS, "focal neurological and cardio-respiratory symptoms" are present, and bubble formation in the inner ear causes vertigo and loss of hearing.

Petri and Andric (2003, p. 29) note that we should qualify reports of a low incidence of DCS in the diving community by the probability that statistics for DCS frequency are skewed by the tendency of divers "to tolerate (ie. tough-out) minor symptoms." Irrespective of incidence, DCS matters, because, as Vann, Butler, Mitchell and Moon (2011) argue, it can cause "permanent invalidism" and "fatal outcomes."

The conclusion of Jankowski, Tikuisis and Nishi (2004, p. 494) was that "moderate, intermittent exercise during decompression," within the ascent phase of diving, offers a potentially economical and practical procedure to reduce VGE and enhance diving safety.

\section{Diving and Divers' Health}

In the literature of Gronning and Aarli (2011) deep diving is diving to depths of more than $50 \mathrm{~m}$ in water. Such diving has a long history. Vaernes (2007) notes that diving techniques have been around since antiquity. He found evidence for this in an archeological dig in Mesopotamia, dating from 4500 B.C. and, in the scholarship of Aristotle, who mentioned a diving machine. From this history, Vaernes (2007, p. 184) traces five historical stages in the development of diving:

1) Breath-hold diving, also called free diving.

2) Diving bells.

3) Surface-supported or helmet diving, also called hard-hat diving.

4) Self-contained underwater breathing apparatus (scuba diving).

5) Saturation diving.

Germonpre (2006) identifies three categories of divers: 1) recreational divers, 2) occupational or commercial divers, and 3) professional divers, a category in which military divers would fit. Gronning and Aarli (2011, p. 18) explain that during diving, several environmental factors impact all divers: "depth, time, temperature and the physical and chemical properties of the gas mixtures employed [in breathing gas]." The subjects in the study by Jankowski, Tikuisis and Nishi were thirty 
nine men who are representative of commercial and professional divers: twenty eight were commercial diving students; eleven were Canadian military divers.

In the literature of Elliott (2004, p. 533) commercial and military divers perform in a "unique workplace." Commercial divers are employed in diverse activities which involve scuba, hosesupplied and bell diving in tasks involving construction, inspection, exploration for minerals, and repair and maintenance of deep water structures and mountain dams. Military divers perform in "unusual conditions." According to Gronning and Aarli (2011), in deep diving, all divers are in an environment that, on its own, can upset their physiology and well-being. Elaborating on this hazardous environment, Benton and Glover (2005, p.240) point out that "the primary challenge to a diver is immersion in a liquid medium" which "introduces hazards such as drowning, hypothermia, and physical exhaustion." Elliott (2004, p.533) argues that all divers must be "medically, mentally, and physically fit to perform their varied tasks," and "the primary objective" of a diving medical is to maximise the diver's safety underwater.

The physician in charge of the diving medical is responsible for certification of a diver's compatibility with relevant health criteria. The examining physician should seek for divers to be in optimal comprehensive health, because, as Vaernes (2007, p.184) points out, reiterating a theme in studies of the diving environment, diving is "potentially dangerous." This danger is due largely to exposure to extreme abnormal pressure, hyperbaric conditions.

The conventional measurement of pressure is bars: 1 bar is normal atmospheric pressure. According to Benton and Glover (2005, p.240) water is much more dense than air is and ambient pressure changes more rapidly with depth than with altitude: "it is necessary to climb a mountain $5,486 \mathrm{~m}$ high to reduce ambient pressure by 0.5 bar $(0.05 \mathrm{MPa})$ but the diver has only to descend $5 \mathrm{~m}$ in water to experience a pressure increase of 0.5 bar $(0.05 \mathrm{MPa}) . "$ Big changes in pressure for relatively small movements in depth are "unique to the diving environment" and are a prominent part of the explanation for diving disorders. Collectively, these disorders are called dysbarism. Another hazard of depth for divers is gas toxicity.

According to Risdall and Gradwell (2011) many investigations have led to a consensus that gases, which are benign or inert at the surface of bodies of water such as the sea, tend to become toxic at depth. Nitrogen, for instance, is prone to narcosis at depth. At increased partial pressures nitrogen behaves as a narcotic stimulant: it produces symptoms which resemble alcohol intoxication, reduced manual adeptness, and diminished mental function. The underlying pathology for these impairments is attributed to an effect of gas on cell membranes.

Oxygen toxicity plays out in two forms, central nervous system (CNS) and pulmonary. The trigger for CNS oxygen toxicity is breathing of oxygen at a partial pressure of 1.50 bars $(150 \mathrm{kPa})$ above the lower limit for toxicity which is 0.3 bar $(30 \mathrm{kPa})$. The classic warning sign of CNS oxygen toxicity is an abrupt epileptiform convulsion.

Pulmonary oxygen toxicity is related to the duration of breathing the gas and the amount of gas intake. In general, the starting point for pulmonary oxygen toxicity is exposure to oxygen above 0.5 bar $(50 \mathrm{kPa})$ for more than twelve hours. Leading symptomatic signals include dry cough, tracheal irritation, and lessening of vital capacity. The third of these signals contributes to the accumulation of gas bubbles in the body as the performance of the lungs declines. The pathology for pulmonary oxygen toxicity is held to be disruption of the endothelial and epithelial cells, respectively, cells in the blood vessels and glandular cells.

Gas bubbles in the body of divers have been implicated in a range of health disorders, including neurological disorders. According to Gronning and Aarli (2011) potential immediate neurological effects of deep diving include hyperoxia, hypoxia, hypercapnia, nitrogen narcosis, and high pressure nervous syndrome. Koshi, Katoh, Abe and Okudera (2000) reported the case of two breath-hold divers who were examined for neurological disorders. Magnetic resonance images (MRI) showed T2weighted hyperintensities in their brain consistent 
with their neurological symptoms. And lesions in their brain suggested cerebral infarction, which physicians attributed to repetitive breath-hold dives. A strong impulse for this conclusion by the researchers was the prior health status of theses divers: these divers were free of vascular diseases and risk factors for stroke with the exception of smoking.

\section{Issues of concern in a medical assessment of divers}

Relying heavily on the scholarship of Bennett, Cronje and Campbell (2006), this section sketches some of the standard medical conditions and disorders that should rank high among the concerns of diving physicians, during a physical assessment of divers. These issues are important because of their potential impact on the safety and performance of divers underwater.

\section{Blood issues}

Anemia: According to Bennett, Cronje and Campbell (2006, p.98), anemia reduces hemoglobin content, a phenomenon which impairs oxygen content to a degree beyond compensation by arterial oxygen. This is a grave scenario, since normal oxygen content is vital to the survival of tissues during the diffusion of gas in the diver's body. The National Oceanic and Atmospheric Administration (NOAA) (as cited in Bennett, Cronje and Campbell, 2006, p.98) deems anemia "relatively disqualifying" to divers.

Bleeding disorders: Individuals with this condition should not dive, because it is a risk factor for "barotrauma damage to air filled spaces such as the sinuses, ears, or lungs from the bleeding that can occur in these organs" (Bennett, Cronje, and Campbell, 2006, p. 98).

Leukemia: This condition, severe malignancy of the white blood cells, rules out diving, because the chemotherapy treatment which it requires has a suppression effect on the immune system: "One should not dive while there are any effects of...drugs on the immune system due to the possibility of overwhelming infection form marine organisms" (Bennett, Cronje and Campbell, 2006, p.101)

\section{Cardiovascular issues}

Germonpre (2006) advises that the cardiovascular implications of diving should be taken very seriously. In this perspective, diving physicians should scrutinise individuals for signs of aortic aneurism, blood disorders, and cholesterol.

Aneurism: This condition involves the dilation and rupture of the aorta, the largest body artery and a critical vessel for the transport of blood from the heart to other areas of the body. Sudden elevations of blood pressure, which are a risk in diving, as in all strenuous sports activities, can trigger an aneurism.

Blood pressure: Ideally, divers should maintain blood pressure levels close to, or below a systolic pressure of $120 \mathrm{~mm} \mathrm{Hg}$. during heart contraction and a diastolic pressure of $80 \mathrm{~mm} \mathrm{Hg}$. during heart relaxation. Abnormally high elevation of blood pressure is a risk factor for several events that can be baleful to divers: stroke due to rupture of brain blood vessels (hemorrhagic stroke), eye problems, and coronary artery disease or heart attack, to name a few of these adverse events.

Cholesterol: Divers should be tested to calculate their balance between 'good' and 'bad' forms of cholesterol, respectively high density lipoprotein cholesterol (HDL) and low density lipoprotein cholesterol (LDL). Divers should also be tested to detect whether a third form of cholesterol fat, triglycerides are present in safe amounts. According to Bennett, Cronje, and Campbell (2006, p. 107) cholesterol should be controlled to reduce the risk of cardiovascular disease: "If unaddressed, cholesterol problems will lead to early and frequently sudden death that may be precipitated by the exercise of diving."

\section{Respiratory Issues}

The respiratory health of divers is a major concern, because, as Moon and Longphre (2006, p.21) point out "Humans are poorly adapted to the underwater environment." In agreement, Pochekutova and Korenbaum (2011, p. 334) note that "Diving activity is connected with a whole set of unfavorable factors on the respiratory system." Today, compressed gas technology supports diving, 
but its agency is not fool proof, another point in the literature of Moon and Longphre (2006, p.21):

Using compressed gas technology humans can breathe underwater, although with effects on pulmonary statics, dynamics, and gas exchange. Breathing compressed gas causes an increase in density leading to impairment of both inspiratory and expiratory flow, and hence a reduction in maximum exercise ventilation.

Against this background, the diving medical should question the individual to uncover any history of asthma, chronic obstructive pulmonary disease (COPD), and chest injuries.

Asthma: This lung disorder degrades breathing capacity. Thus, asthmatic divers may be at a disadvantage in exhaling air during the ascent phase of diving. Additionally, they may be predisposed to pulmonary barotraumas, which, in turn, can induce arterial gas embolism. Notwithstanding these potential hazards, Bennett, Cornje and Campbell (2006, p.190) argue that "The risk of diving is acceptable if the diving candidate with some asthmatic 'history' demonstrates normal pulmonary function at rest and this does not deteriorate with strenuous exercise." They recommend that the asthmatic history diver be free of respiratory disorder symptoms before each dive.

COPD: The characteristic of this condition is chronically inflamed bronchi. Commonly, smoking and polluted air stimulate COPD, which is marked by shortness of breath and heavy mucous production. According to Bennett, Cronje and Campbell (2006, p. 190) "The lungs of an individual with COPD never return to normal. Thus, the diver candidate with COPD may have an increased theoretical risk of burst lung from rupture of obstructed small airways at all times."

Chest injuries: This body damage may cause breathing impairment and concomitant diminished exercise tolerance. In the literature of Bennett, Cronje and Campbell (2006, p. 191), a noteworthy passage on the relevance of chest injuries to diving informs as follows:

Chest injuries may result in scar tissue that makes the lungs vulnerable to tearing and may result in arterial gas embolism, even without causing a pneumothorax. Previous injury with significant scarring, and, where the lungs stick to the chest wall, can lead to air trapping and pulmonary barotraumas with resultant pneumothorax, subcutaneous emphysema, and possibly arterial gas embolism

\section{Other issues}

Alcohol: Bennett, Cronje and Campbell (2006, p.208) say directly: "Alcohol and diving are not compatible." They add that "degraded performance" has appeared in some divers with blood alcohol levels well below the standard of $0.08 \%$ which restricts motor vehicle driving. In their study, Taylor, O'Toole and Ryan (2002) found that alcohol affects diving safety through dehydration and heightened risk of DCS.

\section{Diabetes}

Traditionally, the medical community has considered diabetes a contraindication for deep diving activity. This outlook is based on concern about the depletion of blood glucose level, a condition that impairs physical fitness. Its extreme form, hypoglycemia can occur spontaneously and trigger non-cognitive changes, in which confusion and drowsiness can evolve into loss of consciousness and coma.

\section{The Sekulic and Tocilj study}

The investigation by Sekulic and Tocilj (2006) was concerned primarily with one of the major diving health issues, smoking habit. Sekulic and Tocilj studied a sample of military divers, because, in their view, diving is more demanding in the military community than it is for commercial and recreational divers. The researchers investigated pulmonary function (PF), the respiratory capacity of the lungs, a physiological phenomenon, which is critical for respiration during diving phases and is implicated in pulmonary oxygen toxicity. Their interest was aroused by the scarcity of studies on PF in military divers. Their subjects were male and female, smokers and non-smokers, Croation military divers with 3 to 4 years diving experience. 
A main objective of their investigation was to identify differences in the absolute and relative $\mathrm{PF}$ values in the subjects regarding their smoking status.

The flow volume curves plotted in this study involved the following phases of breathing:

1) Inspiratory vital capacity (VCIN).

2) Forced vital capacity (FVC).

3) Forced expiratory volume in 1 second (FEV1).

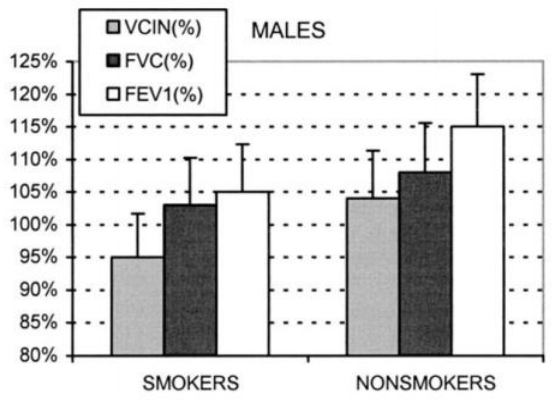

Fig. 1. Graphical presentation of the LAV differences regarding smoking status (values presented are mean $+\mathrm{SD}$ ) males.

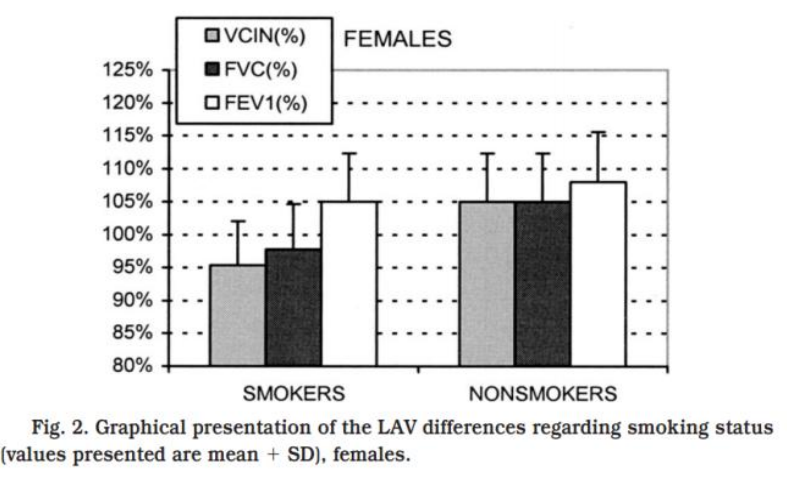

In their findings, the researchers noted that without segregating male and female subjects, there was no significant PF disparity between smokers and nonsmokers on single variable differences. This finding, as the researchers comment, was surprising, given the reputation of smoking as a major "receding factor" in PF (p. 1073).

The researchers argue that the best explanation for the more or less common PF in the subjects was their common experience of rigorous physical fitness training (PFT), which included weight training, aerobic and anaerobic endurance training, running, swimming, and cardio training. The last exercise strengthens the heart, lungs and capacity of the lungs.
The researchers concluded that regular PFT can moderate negative effects of smoking on PF; therefore, regular PFT should be a component of PF improvement therapy.

\section{Discussion}

There is room for improvement in our knowledge of diving health. Verma (2012) has argued that this topic is inadequately recognised, even in the World Health Organisation's International Classification of Diseases list, which is a global gold standard for epidemiological initiatives and health management. Through the two studies it has reviewed and the information it has cited from the diving and diving health literature, this clinical review is a contribution to repairing this defect.

\section{References}

1. Bennett, P., Cronje, F. And Campbell, E. (2006). Assessment of Diving Fitness For Scuba Divers And Instructors. Flagstaff, AZ: Best Publishing Company

2. Benton, P. and Glover, M. (2005). Diving medicine. Travel Medicine and Infectious Disease (2006) 4, 238-254. Retrieved 8 June 2015 from Elsevier Ltd. doi:10.1016/j.tmiad.2005.06.014

3. Daniels, S., Evans, A., Hennessy, T. and Estaugh, K. (1984). Ultrasonic monitoring of Decompression procedures [and discussions]. Philosophical Transactions of the Royal Society of London Series B, Biological Sciences, 304 (1118), Diving and Life atHigh Pressures, pp.153-175. Retrieved 26 May 2015 from http://www.jstor.org/stable/2396162

4. D' Aouts, B., Smith, K., Swanson, H., White, R., Harvey, C., Hunter, W. et al. (1977). Venous gas bubbles: Production by transient, deep isobaric counter diffusion ofhelium against nitrogen. Science, New Series, 197 (4306) 889-891.Retrieved 28 May 2015 from http://www.jstor.org/stable/1744476

5. De Gorordo, A., Vallejo-Manzur, F., Chanin, K. and Varon, J. (2003). Diving 
emergencies.Resuscitation (59) (2003) 171180. Retrieved 10 June 2015from Elsevier Ireland Ltd. doi:10.1016/s0300-9572 (03) 00236-3

6. Elliott, D. (2004). Medical evaluation of working divers in Bove and Davis' Diving Medicine $\left(4^{\text {th }}\right.$ ed.), (Alfred Bove Ed.), pp.533-545.Retrieved 28 May 2015 from http://www.sciencedirect.com

7. Germonpre, P. (2006). The medical risks of underwater diving and their control. International Sportmed Journal, 7 (1), 1-15. Retrieved 27 May 2015 fromAcademic Search Premier

8. Gronning, M. and Aarli, J. (2011). Neurological effects of deep diving.Journal of the Neurological Sciences, 304 (2011), 17-21 Retrieved 27 May 2015 from www.elsevier.com/locate/jns

9. Jankowski, L., Tikuisis, P. and Nishi, R. (2004). Exercise effects during diving and decompression on postdive venous gas emboli. Aviation, Space, and Environmental Medicine 75 (6), 489-496

10. Koshi, K., Katoh, T. Abe, H. And Okudera, T. (2000). Neurological accidents caused by repetitive breath-hold dives: two case reports. Journal of the Neurological Sciences 178 (2000) 66-69. Retrieved 7 June 2015 from Elsevier Science B.V.PII:S0022-510X (00) 00360-9

11. Moon, R. and Longphre, J. (2006). Diving in Encyclopedia of Respiratory Medicine (Geoffrey Laurent and Shapiro, S. Eds.), pp.21-29Retrieved 25 May 2015 from http://www.sciencedirect.com

12. Petri, N. and Andric, D. (2003). Differential diagnostic problems of decompression sickness-Examples from specialist physicians' practices in diving medicine.Archives of Medical Research, 34 (2003), 26-30.Retrieved 28 May 2015 fromwww.elsevier.com/locate/jns

13. Pochekutova, I. and Korenbaum, V. (2011). Acoustic estimation of the impact of a single dive using a closed-type breathing apparatus on the ventilator function of the human lungs.Human Physiology, 37 (3), 334-338. Retrieved $11 \quad$ June 2015 from DOI:10:1134/s0362119711020162

14. Risdall, J. and Gradwell, D. (2011). Extremes of barometric pressure. Anaesthesia And Intensive Care Medicine, 12 (11), 496500Elsevier Ltd.

15. Sekulic, D. and Tocilj, J. (2006). Pulmonary function in military divers: Smoking habitsand physical fitness training influence. Military Medicine, 171, 11:1071

16. Strauss, M. and Borer, R. (2001). Diving medicine: Contemporary topics and their controversies. American Journal of Emergency Medicine 19 (3), 232-238 Retrieved 9 June 2015 from Doi:10.1053/ajem.2001.22654

17. Taylor, D., O'Toole, K. and Ryan, C. (2002). Experienced, recreational scuba divers in Australia continue to dive despite medical contraindications.Wilderness and Environmental Medicine, 13 (2002), 187193Retrieved 10 June 2015 from http:// www.ncbi.nlm.nih.gov/pubmed/12353595

18. Vann, R., Butler, F., Mitchell, S. and Moon, R. (2011). Decompression illness.The Lancet, 2010 (377), 153-164

19. Vaernes, R. (2007). Pressure, effects of extreme high and low in Encyclopedia of Stress ( $2^{\text {nd }}$ ed.), (George Fink et al. Eds.), pp. 184-194Retrieved 26 May 2015 from http://dx.doi.2048/10.1016/B978-

012373947-6-00305-6

20. Verma, R. (2012). International classification of diseases and diving illnesses. MJAFI 68 (1), 61-62. Retrieved 6 June 2015 from doi:10.1016/s0377-1237 (11) 60142-1 